Thorsten R. C. Johnson

Konstantin Nikolaou

Alexander Becker

Alexander W. Leber

Carsten Rist

Bernd J. Wintersperger

Maximilian F. Reiser

Christoph R. Becker

\section{Dual-source CT for chest pain assessment}

viewed for further diagnoses. Technical limitations were insufficient contrast opacification in six and artifacts from respiration in three patients. The most frequent diagnoses were coronary stenoses, valvular and myocardial disease, pulmonary embolism, aortic aneurysm and dissection. Overall sensitivity for the identification of the cause of chest pain was $98 \%$. Correlation to invasive coronary angiography showed $100 \%$ sensitivity and negative predictive value for coronary stenoses. Dual-source CT offers a comprehensive, robust and fast chest pain assessment.

Keywords Chest pain . CT angiography $\cdot$ Coronary artery disease - Pulmonary $\cdot$ Embolism . Aortic dissection

\section{Introduction}

Comprehensive CT angiography protocols for a complete assessment of the thoracic vessels, often referred to as "triple rule out" protocols, are used in the differential diagnosis of chest pain increasingly often [1-4]. These protocols aim to opacify pulmonary and coronary arteries as well as the aorta simultaneously to rule out pulmonary embolism, coronary artery disease and aortic aneurysm or dissection in a single exam. With ECG gating, the acquired images of the coronary arteries should have a similar diagnostic accuracy as a specific coronary $\mathrm{CT}$ angiography. Quite a few studies have meanwhile shown the feasibility of a simultaneous evaluation of these vascular territories in one single breathhold scan with a good sensitivity in the identification of the cause of chest pain [5]. Also, recent studies indicate that coronary CT angiography can be helpful for a fast and cost-effective triage of chest pain patients $[6,7]$. So far, a major limitation of these studies especially in acutely ill patients is the restricted image quality of the coronary arteries in high heart rates [8]. The administration of beta-blockers to lower heart rates is general practice in 16- and 64-slice CT [9]. However, this approach is time consuming and limited by contraindications, and a sufficient reduction of the heart rate cannot be achieved in many acutely ill patients. Initial studies of dualsource CT (DSCT) cardiac imaging have shown a robust image quality and a very good diagnostic accuracy of coronary CT angiography even in high heart rates $[10,11]$. Additionally, a more comprehensive cardiac assessment including wall motion and valve function is possible with DSCT [10]. The aim of this study was to assess the 
diagnostic accuracy of a specific dual-source CT protocol for chest pain assessment, regarding a 6-month follow-up for other findings and coronary angiography in patients in whom coronary artery disease could not be reliably excluded as standard of reference.

\section{Materials and methods}

\section{Patients}

One hundred nine consecutive patients [31 women, 78 men; median age 64 (59-67) years] were prospectively enrolled in the trial. The study was approved by the institutional review board, and informed written consent was obtained from every patient prior to the examination. All patients with acute chest pain were eligible for the study if referred by a colleague after initial diagnostic workup including physical examination, ECG and serum levels of creatinine and TSH. Exclusion criteria were positive ECG changes or troponine test, severe ventricular arrhythmia, a history of severe allergoid reaction to iodinated contrast material, renal insufficiency and young age below 30 years. Also, severe dyspnea with inability to hold the breath for at least $15 \mathrm{~s}$ was regarded as exclusion criterion. The patients were asked to hold their breath for approximately $15 \mathrm{~s}$ prior to the examination. If the breathhold could not be maintained, the patient was excluded from the study, and seven otherwise eligible patients had to be excluded for this reason. Heart rates were $68 \pm 14$, ranging from 58 to $118 \mathrm{bpm}$. Beta-blockers were not administered in preparation of the scan. Eighteen patients had known coronary artery disease and 14 were on continuous oral beta blocker medication. One patient had a known chronic aortic dissection.

\section{Examination}

Examinations were acquired on a DSCT Somatom Definition (Siemens, Forchheim, Germany) in supine position. A coronal topogram was scanned to plan the spiral acquisition, which included the whole chest from the first ribs to the diaphragm. The level of the carina was defined as the trigger point where the second tube is switched on additionally. The volume and flow rate of the contrast material (Ultravist 370, Schering, Germany) were adapted to the patient's body weight as described elsewhere [5] according to Table 1. A bolus tracking technique was used to assess the transit time of the contrast agent. For this purpose, a region of interest was defined in the ascending aorta to detect the arrival of the contrast material bolus in images scanned at 2-s intervals, and the scan was initiated with a delay of $4 \mathrm{~s}$ if the density increased more than 100 Hounsfield units (HU). The patient was then instructed to hold his breath at a mild inspiratory position, and the spiral scan was started. The parameters for the acquisition in craniocaudal direction were 0.33 -s gantry rotation time, $120-\mathrm{kVp}$ tube voltage, $560-\mathrm{mAs}$ effective tube current with $0.6-\mathrm{mm}$ collimation and double Z-sampling. The pitch and the tube current modulation were adapted automatically to the heart rate of the patient. Pitch values ranged between 0.2 and 0.48 , whereas the full tube current was only applied at $70 \%$ of the cardiac cycle for heart rates below $70 \mathrm{bpm}$ and between 30 and $70 \%$ for higher heart rates. Scan duration was $15 \pm 3 \mathrm{~s}$.

\section{Image reconstruction}

Continuous ECG-gated axial slices and coronal images of the whole chest were reconstructed with a temporal resolution of $330 \mathrm{~ms}$ using a soft kernel (B30f). Slice thickness of $3 \mathrm{~mm}$ and $2.5-\mathrm{mm}$ increment were applied to avoid artificial inhomogenities in the lung parenchyma due to undersampling with gating. Additionally, axial slices of the heart were reconstructed for a limited range from the carina to the diaphragm with a $200-\mathrm{mm}$ field of view, $0.75-\mathrm{mm}$ slice thickness and $0.5-\mathrm{mm}$ increment using a B26f convolution kernel. If stents had been implanted, an additional reconstruction was performed using a B46 kernel to reduce blooming and enhance the depiction of the lumen. For ECG gating, a single segment reconstruction algorithm utilizing quarter scan segments from both detectors was applied to achieve a temporal resolution of $83 \mathrm{~ms}$, and delays were set to $300 \mathrm{~ms}$ and $70 \%$ of the cardiac cycle for the initial reconstructions. Also, axial slices with 1-mm thickness and increment were reconstructed with a B26f kernel for the whole cardiac cycle at $10 \%$ intervals for dynamic evaluation.

Table 1 Body-weight-adapted contrast material injection protocol

\begin{tabular}{lll}
\hline Weight $(\mathrm{kg})$ & Volume $(\mathrm{ml})$ & Flow $(\mathrm{ml} / \mathrm{s})$ \\
\hline 50 & 106 & 3.5 \\
55 & 111 & 3.7 \\
60 & 116 & 3.9 \\
65 & 121 & 4.0 \\
70 & 125 & 4.2 \\
75 & 130 & 4.3 \\
80 & 134 & 4.5 \\
85 & 138 & 4.6 \\
90 & 142 & 4.7 \\
95 & 146 & 4.9 \\
100 & 150 & 5.0 \\
105 & 154 & 5.1 \\
110 & 157 & 5.2 \\
115 & 161 & 5.4 \\
120 & 164 & 5.5 \\
\hline
\end{tabular}




\section{Diagnostic evaluation}

The examinations were read by two radiologists in consensus immediately after the exam, including a review of axial and coronal slices for evaluation of pulmonary embolism and aortic disease (Figs. 3 and 4). Additionally, a complete cardiac workup (Figs. 1 and 2) was done using a 3D workstation (Multimodality Workplace, Siemens, Forchheim, Germany) to interactively browse multiplanar reconstructions and volume-rendered images if necessary. Multiphase reconstructions were reviewed as cine loops of the heart with interactive browsing of dynamic short- and long-axis sections. The coronary artery tree was extracted using region-growing algorithms, and curved multi-planar reformats were created for review (Fig. 1a,b). In case of motion artifacts, additional reconstructions with an optimized delay were available. If these still did not resolve motion artifacts sufficiently to make a sufficient diagnostic evaluation feasible, the depiction of the respective coronary artery segment was rated as non-diagnostic and excluded from further analysis. Additionally, the multiphase reconstructions were reviewed to assess wall motion abnormalities and valvular disease (Fig. 2a-d).

Indication for invasive angiography was evaluated by a cardiologist based on CT and clinical findings. Invasive angiography was always initiated if significant coronary artery disease could not be reliably excluded in CT and there was no other evident pathology explaining the
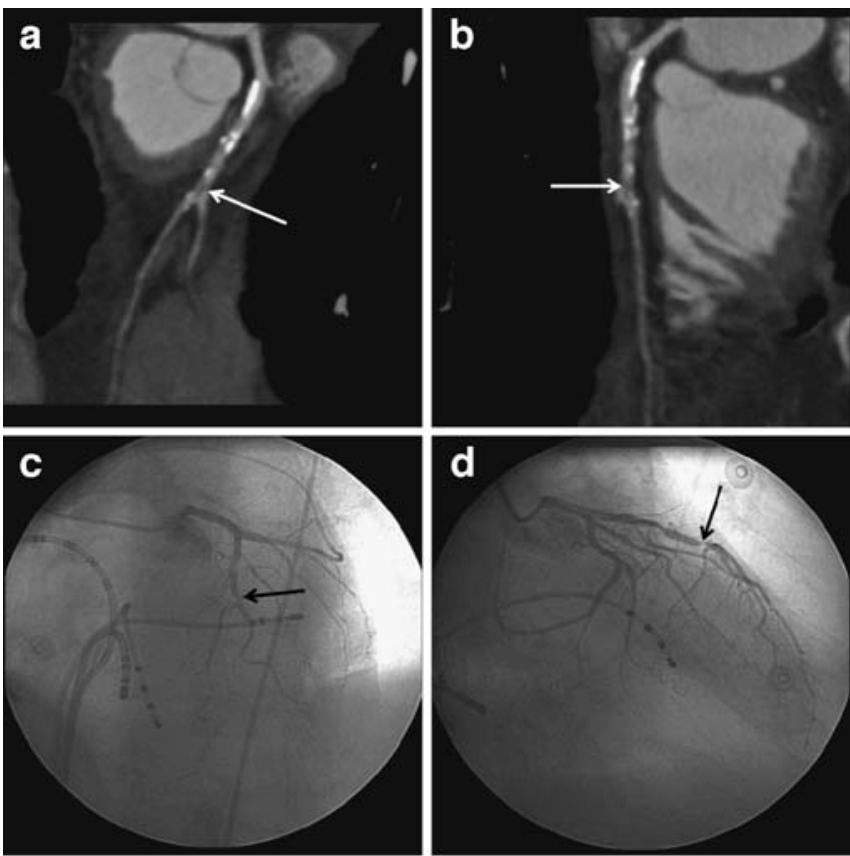

Fig. 1 High grade stenosis (arrows) of the left anterior descending coronary artery. a Curved multiplanar reconstruction along the centerline of the vessel. b Orthogonal curved multiplanar reconstruction. c Left anterior oblique-cranial projection at invasive angiography. d Respective right anterior oblique projection
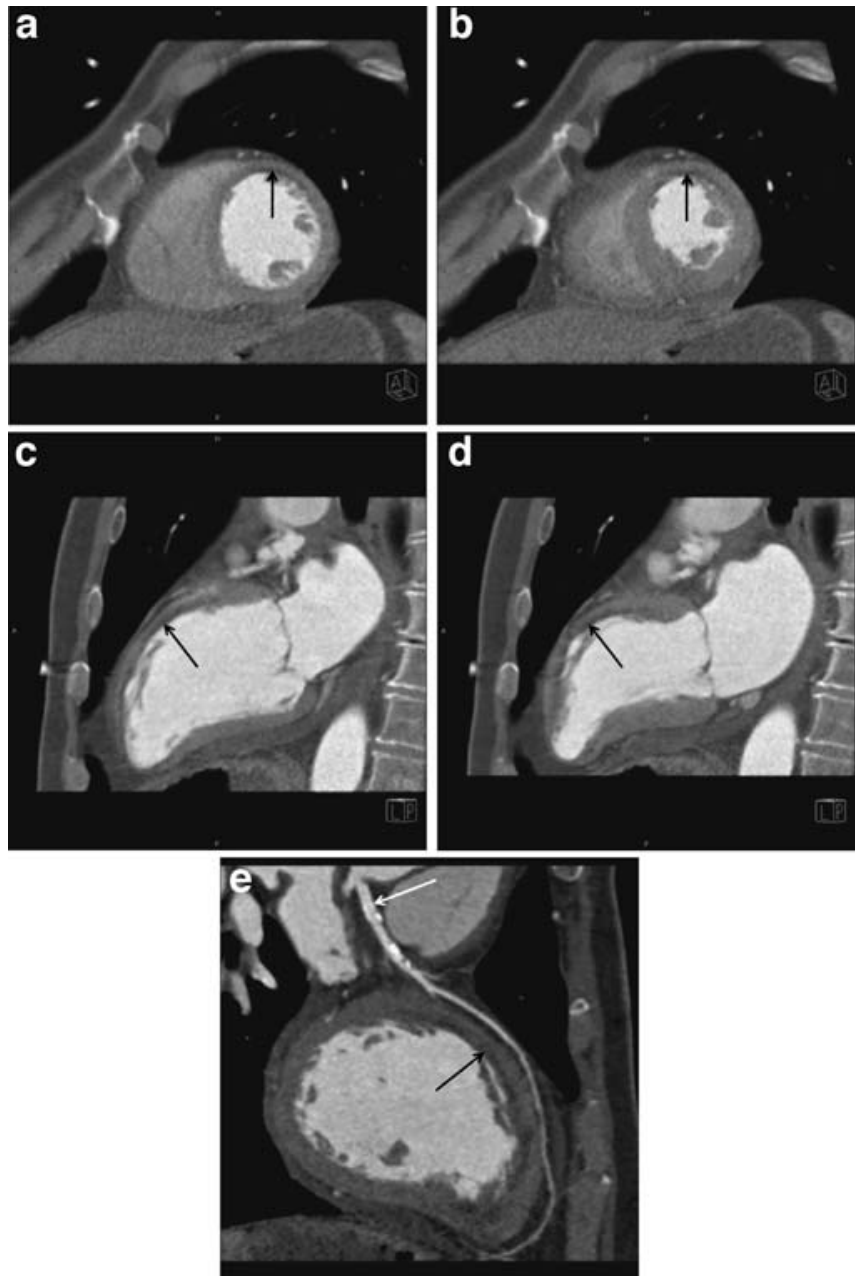

Fig. 2 Subendocardial scar (arrows) from a myocardial infarction in the territory of the left anterior descending coronary artery. a Diastolic mid-ventricular short axis view. b Systolic short axis view. c Diastolic left two-chamber view. d Systolic two-chamber view. Note the dyskinesia bulging out of the anterior wall. e Multiplanar reconstruction of the left anterior descending coronary artery. Note the stent (white arrow) in the proximal vessel, which had been implanted after interventional re-perfusion of the occluded vessel

symptoms. Selective catheterization and coronary angiography were performed by an experienced cardiologist using a transfemoral arterial approach and standard Seldinger technique. At least three standard projections including a $45^{\circ}$ left anterior oblique, a $30^{\circ}$ right anterior oblique and a $45^{\circ}$ left anterior and $30^{\circ}$ cranial projection were acquired for both coronary arteries. For quantitative coronary assessment (QCA) analysis, a densitometrical analysis of two projection images was performed using the Quant-Cor QCA software (Siemens, Erlangen). Conventional coronary angiograms of 29 patients were available for correlation. A third reader correlated the findings in the reports to invasive coronary angiography and performed dose calculations, which were based on the dose length product given in the patient protocol of the CT scanner. Also, he 
reviewed the files of patients with initially negative results for further diagnoses in the subsequent 6 months. The readers of the initial CT images were unaware of any findings from conventional angiography, and most invasive angiographies were performed to further evaluate or treat lesions detected in CT. Thus, the cardiologists performing the coronary angiography were mostly aware of the CT findings.

\section{Statistical analysis}

Continuous variables are given as median and range. Ninety-five percent confidence intervals were calculated using MedCalc software (MedCalc Software, Mariakerke, Belgium). Diagnostic accuracy was calculated as sensitivity, specificity, positive and negative predictive values based on standard contingency tables.

\section{Results}

\section{Technical success}

The scans were acquired from all 109 patients without severe adverse events. Although previously tested, three patients were unable to hold their breath for the duration of the scan, which resulted in artifacts in the lower part of the chest, rendering the coverage and depiction of the coronary arteries insufficient for diagnostic evaluation. All examinations showed an adequate contrast enhancement of the pulmonary arteries, the coronary arteries and the aorta except for six examinations. In two, the bolus was too short due to hemodynamic parameters, making the assessment of pulmonary embolism only possible in the central arteries. In three examinations an erroneous administration of a smaller volume of contrast material caused a weak opacification of the pulmonary arteries, and in one examination overall opacification was too weak (220$245 \mathrm{HU})$ for coronary artery assessment.

\section{Patient findings}

The diagnoses in the patient population were spread over the different organs and vascular territories quite evenly (Table 2). Ten patients had pulmonary embolism (Fig. 3) and three had morphological signs of chronic pulmonary hypertension. Twelve patients showed other, non-vascular pulmonary findings including pneumonic consolidation in four patients and edema from left heart failure in four cases. Thirty-seven patients had coronary atherosclerosis, and in 21 of these patients, stenoses were rated as hemodynamically significant $(>50 \%)$ (Fig. 1). Three coronary arteries were occluded. Fifteen patients had had bypass surgery, and five bypass graft vessels were occluded. One bypass was rated as stenotic, but invasive angiography showed a patent lumen and a very slow runoff. There were 20 noncoronary cardiac findings including valvular disease in seven patients. Among those, there were four cases of aortic stenosis, one bicuspid aortic valve and one with vegetations from endocarditis. In one patient an insuffi-
Table 2 Diagnoses found in the study population

\begin{tabular}{|c|c|c|c|c|c|c|}
\hline Territory & & Type & & Diagnosis & & $\mathrm{n}$ \\
\hline \multirow[t]{4}{*}{ Pulmonary } & $19 \%$ & Vascular & $62 \%$ & Embolism & $77 \%$ & 10 \\
\hline & & & & Pulmonary hypertension & $23 \%$ & 3 \\
\hline & & Non-vascular & $38 \%$ & Pneumonia & $50 \%$ & 4 \\
\hline & & & & Edema & $50 \%$ & 4 \\
\hline \multirow[t]{7}{*}{ Cardiac } & $40 \%$ & Coronary & $84 \%$ & Atherosclerosis & $100 \%$ & 37 \\
\hline & & & & Relevant stenosis & $57 \%$ & 21 \\
\hline & & & & Occlusion & $8 \%$ & 3 \\
\hline & & Bypass grafts & $18 \%$ & Patent & $75 \%$ & 15 \\
\hline & & & & Occluded & $25 \%$ & 5 \\
\hline & & Non-coronary & $13 \%$ & Valvular & $50 \%$ & 7 \\
\hline & & & & Myocardial & $50 \%$ & 7 \\
\hline \multirow[t]{3}{*}{ Aortic } & $13 \%$ & & & Aneurysm & $50 \%$ & 7 \\
\hline & & & & Dissection & $43 \%$ & 6 \\
\hline & & & & Rupture & $7 \%$ & 1 \\
\hline \multirow[t]{2}{*}{ Other } & $7 \%$ & Vascular variants & $25 \%$ & & & 2 \\
\hline & & Neoplastic & $75 \%$ & & & 6 \\
\hline Negative & $24 \%$ & & & & & 26 \\
\hline Sum of patients & $100 \%$ & & & & & 109 \\
\hline
\end{tabular}




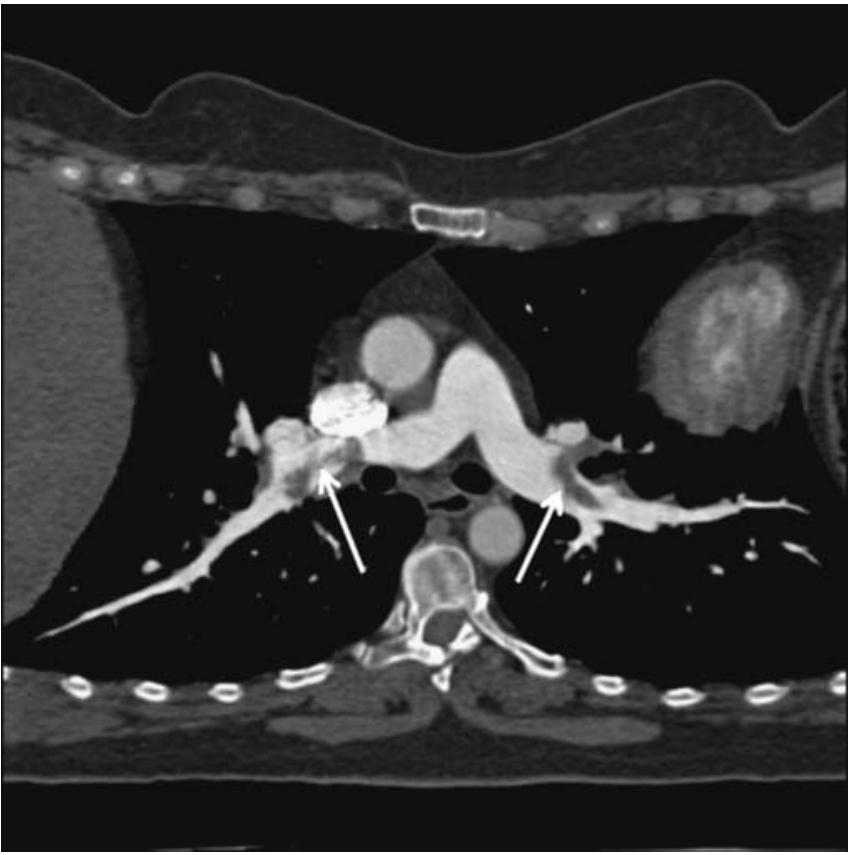

Fig. 3 Curved multiplanar reconstruction of the lower lobe pulmonary arteries showing emboli in the lobar and segmental arteries

ciency of tricuspid valve leaflets was diagnosed. Three patients had had aortic valve replacement, and the valve prostheses worked normally. Seven patients showed an abnormal global or regional wall motion with pulmonary congestion in four cases. Areas of myocardial infarction were noted in three patients (Fig. 2). Other non-coronary cardiac findings were left ventricular hypertrophy, scars from myocarditis and ventricular thrombi. Among the aortic pathologies there were seven aneurysms, five dissections (Fig. 4), one case of intramural hematoma and one acute rupture with active bleeding into the mediastinum. Incidental vascular findings were a lusorian
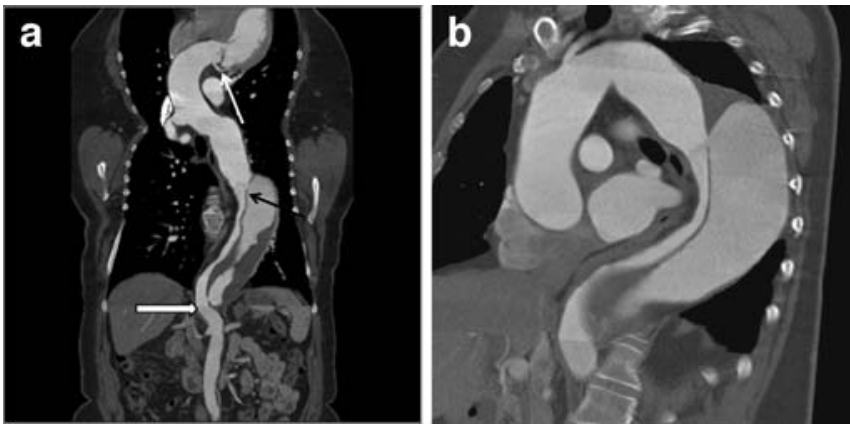

Fig. 4 Dissection of the descending aorta. a Multiplanar reconstruction. Arrows indicate the depiction of the left coronary artery in the gated reconstruction (black arrow), the dissection membrane (white arrow) and the junction between the gated scan and the nongated scan, which had been continued in this case to include the whole extent of the dissection (outlined arrow). b Angulated paracoronal reconstruction showing the topography artery and a right descending aorta with Kommerell's diverticulum (Fig. 5). Another frequent finding was neoplastic lesions, partially with unknown primary cancer. Three patients had disseminated pulmonary metastases, one patient had a previously known lymphoma and one an esophageal cancer. In 26 patients there were no findings that were regarded as the main cause of chest pain. The review of the files of those patients in a 6-month follow-up showed additional diagnoses in two patients: One had intermittent arrhythmia that was identified as the cause of chest pain. The other patient developed pleural effusion and was diagnosed to have pleuritis. In the other 24 patients there was no evidence of the cause of chest pain in followup. Considering those results as correct-negative, the sensitivity of the protocol in the identification of the cause of chest pain amounts to $98 \%$. Specificity can be estimated at $92 \%$ based on these data, although the exact significance of the findings for chest pain is frequently unclear and may therefore be regarded as arbitrary.

\section{Correlation to invasive angiography}

The 29 conventional coronary angiograms that were available for correlation of the coronary findings were evaluated in a segment-based and a patient-based analysis. Thirty-two coronary artery segments (7\%) of these patients were excluded from analysis due to small size or insufficient visualization in CT. Of the 29 patients, 25 had evidence of coronary atherosclerosis in CT. Nineteen stenoses were rated as potentially hemodynamically relevant with more than $50 \%$ stenosis in CTA, but invasive angiography confirmed only 15 of those, whereas the other 4 were overrated. There were no false-negative results, i.e., all the stenoses had been cited in CTA, although it has to be taken into account that the indication for invasive angiography was frequently derived from CTA findings. Thus, sensitivity, specificity, positive and negative predictive
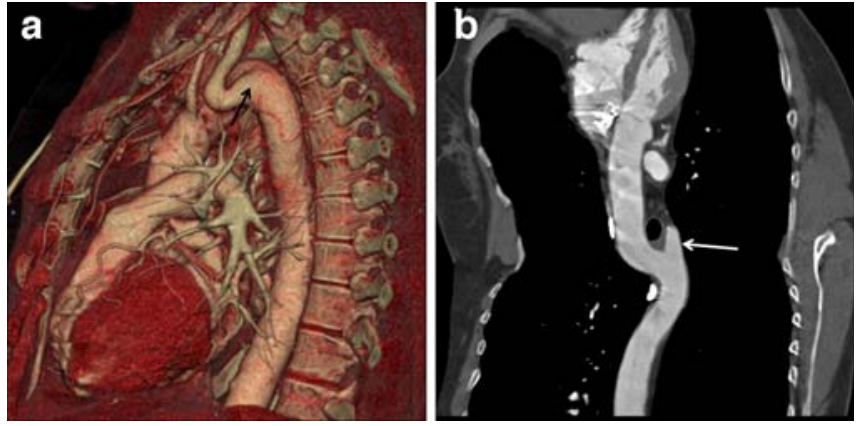

Fig. 5 Kommerell's diverticulum (arrows). a Volume-rendered reconstruction showing the origin from the aortic arch and the course of the subclavian artery. Note the median-right course of the descending aorta ventral to the spine. b Curved multiplanar reconstruction showing the proximity to the trachea 
value amounted to $100 \%, 99 \%, 79 \%$ and $100 \%$, respectively, in the segment-based analysis.

In the patient-based analysis, all 13 patients with significant stenoses were identified. There were two patients with completely negative CTA and symptoms very suggestive of coronary ischemia who had additional invasive angiography, and both of them remained negative. The overrated stenoses occurred in patients with other, significant stenoses. Thus, CTA achieved full diagnostic accuracy in correlation to invasive angiography for this patient-based analysis. The database of the coronary angiography laboratory was screened for those patients who had not initially been examined invasively, and there were no readmissions for angiography within 6 months after inclusion of the last patient.

\section{Radiation exposure}

As the patient protocols had not been archived in all patients, there were only 51 dose-length products available for calculation. Based on a conversion factor of 0.012 for chest exams, the estimated equivalent dose ranged between $9.2 \mathrm{mSv}$ in low heart rates with strict dose modulation to $19.5 \mathrm{mSv}$ in tall patients with heart rates around $70-80$ $\mathrm{bpm}$, which resulted in a wide pulsing interval of $30-70 \%$ with a still quite low pitch below 0.3 . Overall, the median radiation exposure was $15.1 \mathrm{mSv}$ (95\% CI 9.9-18.8).

\section{Discussion}

CT angiography can be regarded as the modality of choice for the assessment of pulmonary embolism and aortic disease in acute care [12]. The fast volume coverage that has become available with recent CT technology has triggered efforts for a combined assessment of these pathologies, and the additional evaluation of the coronary arteries would make the protocol a universal diagnostic tool for chest pain assessment $[2,4,12]$. Quite a few studies showed the feasibility of this approach based on 64-slice CT technology with temporal resolution in the area of 165 $200 \mathrm{~ms}$, but also indicated that coronary CTA still represented the limitation of the method due to the high incidence of high heart rates that made coronary assessment unreliable [1, 3]. Recent studies have shown that diagnostic images of the coronary arteries can be obtained reliably with dual-source CT even in very high heart rates $[10,11]$. Thus, dual-source CT was expected to greatly improve the diagnostic accuracy of the combined CT protocol, and our results indicate that this assumption was correct: There were no examinations rated as nondiagnostic because of blurring of the coronary arteries due to high heart rates. There were six exams with partially insufficient contrast opacification, which mainly affected pulmonary artery evaluation and three examinations with breathing artifacts that impeded coronary assessment, but otherwise the main coronary artery segments were reliably assessable. This is reflected in the detailed analysis of the coronary CT angiography in relation to invasive angiography, in which only $7 \%$ of coronary artery segments were excluded, mainly due to very small size in distal vessel segments and side branches. Regarding the diagnostic accuracy, the excellent performance in the patient-based analysis confirms that the method can be very helpful for fast patient triage. However, it has to be acknowledged that stenoses were quite frequently rated as "potentially relevant' in CTA so that invasive angiography was not only necessary for intervention, but also for definite diagnosis. Also, some false-positive results would have to be expected in larger patient groups.

Still, it has to be emphasized that a valid, relevant diagnosis could be made in $62 \%$ of the patients, and in $25 \%$ relevant diseases were ruled out. Regarding sensitivity for the cause of chest pain, the value of $98 \%$ in the present study is somewhat higher than the $93 \%$ observed in a study on ECG-gated 64-slice thoracic CTA [1] and compares well with an initial DSCT study that reported a sensitivity of $96 \%$ [13]. In $13 \%$ of patients there were findings such as coronary atherosclerosis or vascular variants that were not immediately relevant as a reason for chest pain, but that are still valuable information for further patient management.

Comparing the results of coronary CTA correlation in this study, sensitivity, specificity, positive and negative predictive values of $100 \%, 99 \%, 79 \%$ and $100 \%$ are comparable to an initial study by Scheffel et al. [11]. They observed respective values of $96 \%, 98 \%, 86 \%$ and $99 \%$ for dual-source CT coronary CTA without heart rate control in a high pretest-probability group, although the rate of $1.4 \%$ non-assessable segments was remarkably low compared to $7 \%$ in our study. The somewhat higher negative and lower positive prediction may be attributed to a more sensitive diagnostic reading in our group of acutely ill patients. In a similar 64-slice CT study by Nikolaou et al. [9], respective values of $97 \%, 79 \%, 86 \%$ and $96 \%$ with $10 \%$ nonassessable segments were recorded for an intermediate likelihood population with strict heart rate control. These values suggest that a similar accuracy can be achieved with DSCT without beta blockers.

Due to the variability in body size and habitus, the equivalent doses calculated from dose length products are only correct for less than half of the population and therefore can only serve as rough estimations. The median value of $15.1 \mathrm{mSv}$ is about twice that of coronary artery CTA with dual-source CT, but only moderaltely higher than that for coronary CTA alone with single-source 64-slice CT $[14,15]$. The increase is mainly related to the high heart rates in this acute patient group that require a wider pulsing interval for the reconstruction of systolic images. The longer coverage including the upper chest contributes rather little to the dose because it is acquired normally with only one tube switched on. Therefore, performing coronary 
CTA alone without coverage of the upper chest would save only little of the dose in this patient group. Thus, the additional dose from the longer coverage is well invested considering the diagnostic value. Also, comparing DSCT to single source CTA, the high diagnostic accuracy of DSCT can be regarded as well invested dose compared to single source scanners in which the dose can be estimated to be higher for this patient group because the pitch would have to be reduced to apply multi-segment reconstruction algorithms $[16,17]$, and pulsing would mostly have to be switched off to yield diagnostic examinations with optimized reconstructions [8]. Also, considering alternative diagnostic approaches for the evaluation of chest pain, for example, including pulmonary CTA, aortic CTA and invasive coronary angiography, this combination would result in a higher total patient dose [1]. Magnetic resonance imaging would be an alternative modality for the assessment of pulmonary embolism and aortic pathology, and an MR exam of myocardial wall motion and perfusion could serve as a method to rule out hemodynamically relevant coronary pathology without radiation exposure $[18,19]$. However, the limited availability and the long examination times currently let such an approach seem less applicable in a clinical setting [20].

Several limitations of this study have to be acknowledged. One limitation is the exclusion of patients with the evidence of an acute coronary syndrome based on ECG or serum markers, which causes a bias in the assessment of the diagnostic accuracy of coronary CTA. Another limitation is the lacking correlation to other diagnostic approaches in the evaluation of pulmonary embolism and aortic disease. Of course, additional ventilation/perfusion scintigraphy and aortic MRI would be desirable for study purposes, but cannot be justified considering the necessary time and the encroachment of acutely ill patients and the involved radiation exposure. Also, additional invasive coronary angiography of the patients without coronary findings would be helpful to assess the actual negative predictive value, but the application of this invasive procedure involving radiation exposure to patients with other pathologies would of course not be acceptable. The follow-up of the coronary angiography database that showed no readmissions at least confirms the unlikelihood of relevant coronary artery stenoses in these patients.

The fact that immediately therapy-relevant findings in all vascular districts were represented and quite evenly distributed in our patient population emphasizes that a combined protocol can be a valuable tool for diagnostic workup of acute, unclear chest pain. In conclusion, the specific dual-source CT protocol for chest pain assessment proved to be a very helpful tool offering a fast diagnostic workup and patient triage.

\section{References}

1. Johnson TRC, Nikolaou K, Wintersperger BJ, Knez A, Boekstegers P, Reiser MF, Becker CR (2007) ECG gated 64 slice CT angiography for the differential diagnosis of acute chest pain. AJR Am J Roentgenol 188:76-82

2. Savino G, Herzog C, Costello P, Schoepf UJ (2006) 64 slice cardiovascular CT in the emergency department: concepts and first experiences. Radiol Med (Torino) 111:481-496

3. White CS, Kuo D, Kelemen M, Jain V, Musk A, Zaidi E, Read K, Sliker C, Prasad R (2005) Chest pain evaluation in the emergency department: can MDCT provide a comprehensive evaluation? AJR Am J Roentgenol 185:533-540
4. Raptopoulos VD, Boiselle PB, Michailidis N, Handwerker J, Sabir A, Edlow JA, Pedrosa I, Kruskal JB (2006) MDCT angiography of acute chest pain: evaluation of ECG-gated and nongated techniques. AJR Am J Roentgenol 186:S346-356

5. Johnson TRC, Nikolaou K, Wintersperger BJ, Fink C, Rist C, Leber AW, Knez A, Reiser MF, Becker CR (2007) Optimization of contrast material administration for electrocardiogram-gated computed tomographic angiography of the chest. J Comput Assist Tomogr 31:265-271

6. Hoffmann U, Nagurney JT, Moselewski F, Pena A, Ferencik M, Chae CU, Cury RC, Butler J, Abbara S, Brown DF, Manini A, Nichols JH, Achenbach S, Brady TJ (2006) Coronary multidetector computed tomography in the assessment of patients with acute chest pain. Circulation 114:2251-2260
7. Sato Y, Matsumoto N, Ichikawa M, Kunimasa T, Iida K, Yoda S, Takayama T, Uchiyama T, Saito S, Nagao K, Tanaka H, Inoue F, Furuhashi S, Takahashi M, Koyama Y (2005) Efficacy of multislice computed tomography for the detection of acute coronary syndrome in the emergency department. Circ J 69:1047-1051

8. Leschka S, Wildermuth S, Boehm T, Desbiolles L, Husmann L, Plass A, Koepfli P, Schepis T, Marincek B, Kaufmann PA, Alkadhi H (2006) Noninvasive coronary angiography with 64-section CT: effect of average heart rate and heart rate variability on image quality. Radiology 241:378-385 
9. Nikolaou K, Knez A, Rist C,

Wintersperger BJ, Leber A, Johnson T, Reiser MF, Becker CR (2006) Accuracy of 64-MDCT in the diagnosis of ischemic heart disease. AJR Am J Roentgenol 187:111-117

10. Johnson TR, Nikolaou $K$, Wintersperger BJ, Leber AW, von Ziegler F, Rist C, Buhmann S, Knez A, Reiser MF, Becker CR (2006) Dualsource CT cardiac imaging: initial experience. Eur Radiol 16:1409-1415

11. Scheffel H, Alkadhi H, Plass A, Vachenauer R, Desbiolles L, Gaemperli $\mathrm{O}$, Schepis T, Frauenfelder T, Schertler T, Husmann L, Grunenfelder J, Genoni M, Kaufmann PA, Marincek B, Leschka S (2006) Accuracy of dualsource CT coronary angiography: first experience in a high pre-test probability population without heart rate control. Eur Radiol 16:2739-2747

12. Chiles C, Carr JJ (2005) Vascular diseases of the thorax: evaluation with multidetector CT. Radiol Clin North Am 43:543-569 viii
13. Johnson TR, Nikolaou K, Fink C, Becker A, Knez A, Rist C, Reiser MF, Becker CR (2007) Dual-source CT in chest pain diagnosis. Radiologe 47:301-309

14. Flohr TG, McCollough $\mathrm{CH}$, Bruder $\mathrm{H}$, Petersilka M, Gruber K, Suss C, Grasruck M, Stierstorfer K, Krauss B, Raupach R, Primak AN, Kuttner A, Achenbach S, Becker C, Kopp A, Ohnesorge BM (2006) First performance evaluation of a dual-source CT (DSCT) system. Eur Radiol 16:256-268

15. Hausleiter J, Meyer T, Hadamitzky M, Huber E, Zankl M, Martinoff S, Kastrati A, Schomig A (2006) Radiation dose estimates from cardiac multislice computed tomography in daily practice: impact of different scanning protocols on effective dose estimates. Circulation 113:1305-1310

16. Halliburton SS, Stillman AE, Flohr T, Ohnesorge B, Obuchowski N, Lieber M, Karim W, Kuzmiak SA, Kasper JM, White RD (2003) Do segmented reconstruction algorithms for cardiac multi-slice computed tomography improve image quality? Herz 28:20-31
17. McCollough CH, Primak AN, Saba O, Bruder H, Stierstorfer K, Raupach R, Suess C, Schmidt B, Ohnesorge BM, Flohr TG (2007) Dose performance of a 64-channel dual-source CT scanner. Radiology 243:775-784

18. Gani F, Jain D, Lahiri A (2007) The role of cardiovascular imaging techniques in the assessment of patients with acute chest pain. Nucl Med Commun 28:441-449

19. Kwong RY, Schussheim AE, Rekhraj $\mathrm{S}$, Aletras AH, Geller N, Davis J, Christian TF, Balaban RS, Arai AE (2003) Detecting acute coronary syndrome in the emergency department with cardiac magnetic resonance imaging. Circulation 107:531-537

20. Kumar A, Friedrich MG (2007) Acute chest pain syndrome: will MRI shake up cardiovascular care in the emergency room? Expert Review Of Cardiovascular Therapy 5:139-141 